\title{
Evaluation of latent tuberculosis infection surveillance in Peel region, Ontario, 2010-2014
}

\author{
JA Majerovich ${ }^{1,2}$, L Fernandes², M Varia ${ }^{2 *}$
}

\section{Abstract}

Background: In Canada, identification and treatment of individuals with latent tuberculosis infection (LTBI) is a key component in preventing the progression of LTBI to active tuberculosis (TB). In Peel region, a large municipality in Ontario where half of the population is foreign-born, LTBI surveillance data are also critical to understanding the local epidemiology of TB.

Objective: To evaluate LTBI surveillance data collected through the integrated Public Health Information System (iPHIS) from 2010 to 2014 by assessing data quality and usefulness and to provide recommendations to improve surveillance for Peel region.

Methods: Using the European Centre for Disease Prevention and Control framework for surveillance evaluation, data quality was assessed based on completeness and validity of key variables in the iPHIS database. Usefulness of surveillance data in informing program decisions was assessed through interviews with stakeholders from Peel Public Health.

Results: Of 6,576 iPHIS records evaluated, data for gender and date of birth were greater than $99 \%$ complete, while more than half of the risk factor fields were blank or 'unknown'. A comparison of 192 paper charts to the corresponding iPHIS record identified coding errors in over $40 \%$ of iPHIS risk factor fields. Treatment completion documented in iPHIS (20\%) was lower than data obtained from a follow-up telephone survey of cases (50\%). Stakeholders found surveillance data to be useful (100\%), however, recommendations were made for improvement of data collection and analysis.

Conclusion: Evaluating LTBI surveillance to improve data quality and usefulness for program planning is essential in an era of TB elimination. This evaluation resulted in standardization of data entry processes and continuation of direct follow-up with LTBI clients to confirm treatment completion. Work to understand barriers to treatment initiation and completion is currently underway.

\author{
Affiliations \\ 1 Field Service Training and \\ Response Division, Public Health \\ Agency of Canada, Ottawa, ON \\ ${ }^{2}$ Peel Public Health, Mississauga, \\ $\mathrm{ON}$
}

*Correspondence: monali.varia@ peelregion.ca

Suggested citation: Majerovich JA, Fernandes L, Varia M. Evaluation of latent tuberculosis infection surveillance in Peel region, Ontario, 2010-2014. Can Commun Dis Rep. 2017;43(5):114-8. https://doi.org/10.14745/ ccdr.v43i05a06

\section{Introduction}

Infection with Mycobacterium tuberculosis can result in latent tuberculosis infection (LTBI) or active tuberculosis (TB) (1). The progression of LTBI to active TB can be reduced by up to $90 \%$ with nine months of preventive treatment $(1,2)$. The World Health Organization has identified that better identification and treatment of those with LTBI who are at higher risk of progressing to active TB is integral to the new TB elimination goals (3). Although Canada has a low incidence of TB overall, rates are higher among sub-populations such as immigrants from countries with high incidence of TB, travellers to these countries and Indigenous Canadians. The Region of Peel is a large municipality in Ontario with a population of 1.4 million. Half (50.5\%) of Peel's population is foreign-born, many from TB-endemic countries. This is higher than the percentage of foreign-born individuals in Ontario (28.5\%) (4). In 2014, the age-standardized active TB incidence rate in Peel was 9.1 per 100,000, compared to 4.0 per 100,000 for Ontario (5).
Approximately 1,200 LTBI cases are reported each year to the local health department, Peel Public Health (PPH) (6).

Identification and treatment of individuals with LTBI at increased risk of progressing to active TB is a pillar of the strategy to prevent the progression of LTBI to active TB. Canadian Tuberculosis Standards target at least $80 \%$ of those LTBI cases that start treatment will complete the required number of doses (1). Peel LTBI surveillance data are used to monitor treatment completion, identify population groups at higher risk of active disease and evaluate the effectiveness of program interventions.

Despite the importance of surveillance to evaluate the effectiveness of TB prevention and control efforts, there is limited published evidence evaluating LTBI surveillance (7-9). The objectives of this study were to evaluate LTBI surveillance data collected through Ontario's integrated Public Health Information System (iPHIS) by assessing two surveillance attributes-data 
quality (data completeness and validity) and usefulness, and provide recommendations to improve LTBI surveillance in Peel region.

In Ontario, iPHIS is the database used by all public health departments to report information on cases of reportable diseases (including LTBI) to the Ministry of Health and Long-Term Care, as mandated under Ontario's Health Protection and Promotion Act, R.S.O. $1990(10,11)$. Data on LTBI cases are passively reported to $\mathrm{PPH}$ by local reporting sources such as community clinicians and hospitals. When a case of LTBI is reported to $\mathrm{PPH}$, public health nurses document case information in a paper-based client chart, which is then entered into iPHIS. Surveillance data are routinely analyzed by PPH epidemiologists for programming implications.

\section{Methods}

The evaluation of the LTBI surveillance system was based on the European Centre for Disease Prevention and Control (ECDC) framework (12). This framework was chosen because of its focus on data quality, which was a primary objective of the surveillance evaluation. The surveillance attributes that were assessed are summarized in Table 1. Internal completeness, and internal and external validity were selected because program decisions are based on the information available in iPHIS. Usefulness was chosen to identify surveillance strengths and opportunities for improving the use of data for public health action.

Table 1: Latent tuberculosis infection evaluation surveillance attributes

\begin{tabular}{|l|l|}
\hline \multicolumn{1}{|c|}{ Attribute } & \multicolumn{1}{c|}{ Definition } \\
\hline Internal completeness & $\begin{array}{l}\text { Proportion of complete data fields } \\
\text { within the database }\end{array}$ \\
\hline Internal validity & $\begin{array}{l}\text { Extent of errors within the surveillance } \\
\text { system, e.g. coding errors in translating } \\
\text { from one level of the system to the next }\end{array}$ \\
\hline External validity & $\begin{array}{l}\text { Whether information recorded about } \\
\text { cases is correct when compared to an } \\
\text { external database or 'gold standard' }\end{array}$ \\
\hline Usefulness & $\begin{array}{l}\text { Whether surveillance results are used for } \\
\text { public health action }\end{array}$ \\
\hline
\end{tabular}

Internal completeness: Internal completeness was measured by calculating the proportion of missing (i.e., blank and 'unknown') responses for selected variables. These include: client demographics (date of birth, gender, origin), risk factors (behavioural social factors, exposure settings, medical risk factors) and treatment variables (treatment start date and treatment end date as specified by the client or their physician). Relevant data fields from all Peel LTBI cases reported from 2010 to 2014 were extracted from the iPHIS database using Cognos ReportNet and analyzed using Stata 14.

Internal validity: Using PPH's paper-based charts for LTBI cases as the standard, internal validity was assessed by comparing data in iPHIS with the paper chart for selected variables. Due to the large number of LTBI cases, a sample size of 203 LTBI cases from 2014 was calculated based on a 95\% confidence level, a population size of 1,157 , a hypothesized $20 \%$ frequency of the outcome factor in the population and a design effect of 1.0 for a random sample (www.openepi.com/SampleSize/SSPropor.htm). Every fifth chart from 2014 was sampled and 192 charts were assessed because some of the charts could not be immediately located.

External validity: External validity was assessed by comparing LTBI treatment completion data from 2010 to 2014 in iPHIS to treatment completion data obtained from active telephone follow-up of LTBI cases. The telephone survey of LTBI cases was conducted by PPH staff from July 2015 to April 2016 with cases known to have started treatment. Given the length of treatment for LTBI is typically nine months for the first-line regimen (1), the 208 clients contacted were likely diagnosed with LTBI in 2014 and 2015.

Usefulness: Eight semi-structured interviews were conducted in person with internal stakeholders from $\mathrm{PPH}$ to assess the usefulness of LTBI surveillance for informing public health action. Stakeholders were chosen to represent a range of viewpoints, from frontline public health nurses who are directly involved in the system's operation to public health decision-makers, including the medical officer of health. Stakeholders were asked about actions taken as a result of surveillance data, surveillance challenges and opportunities. Responses were recorded in text format and data analysis was performed by three PPH epidemiologists (JAM, MV and the TB epidemiologist) who first assessed themes individually and then came to consensus regarding common themes using a facilitated small group method.

\section{Results}

Internal completeness: Of 6,576 LTBI cases, data for gender and date of birth were almost $100 \%$ complete (Table 2 ). Of LTBI cases who had missing or 'unknown' origin $(n=1,716), 0.1 \%$ had birth province documented and $1.9 \%$ had immigration birth country documented. Completeness was suboptimal for the data fields for treatment end date (64.6\% incomplete), treatment start date $(52.3 \%$ incomplete) and risk factor (54.7\% incomplete).

\section{Table 2: Internal completeness of latent tuberculosis infection data fields}

\begin{tabular}{|l|r|r|r|r|}
\hline Variable & \multicolumn{1}{|c|}{$\begin{array}{c}\text { Total } \\
\text { number } \\
\text { of } \\
\text { records }\end{array}$} & $\begin{array}{c}\text { Number } \\
\text { (\%) of } \\
\text { records } \\
\text { with } \\
\text { missing } \\
\text { data field }\end{array}$ & $\begin{array}{c}\text { Number } \\
\text { (\%) of } \\
\text { records } \\
\text { with } \\
\text { 'unknown' } \\
\text { data field }\end{array}$ & $\begin{array}{c}\text { Number (\%) } \\
\text { of records } \\
\text { with } \\
\text { complete } \\
\text { data }\end{array}$ \\
\hline $\begin{array}{l}\text { Date of } \\
\text { birth }\end{array}$ & 6,576 & $0(0)$ & $0(0)$ & $6,576(100)$ \\
\hline Gender & 6,576 & $0(0)$ & $46(0.7)$ & $6,530(99.3)$ \\
\hline Origin & 6,576 & $7(0.1)$ & $1,709(26.0)$ & $4,860(73.9)$ \\
\hline Risk factor & 6,576 & $270(4.1)$ & $3,326(50.6)$ & $2,980(45.3)$ \\
\hline $\begin{array}{l}\text { Treatment } \\
\text { start date }\end{array}$ & 6,576 & $3,436(52.3)$ & $0(0)$ & $3,140(47.7)$ \\
\hline $\begin{array}{l}\text { Treatment } \\
\text { end date }\end{array}$ & 3,140 & $2,030(64.6)$ & $0(0)$ & $1,110(35.4)$ \\
\hline
\end{tabular}

Abbreviation: \%, percentage

${ }^{1}$ Calculated using the total number of clients that started LTBI treatment as the denominator 
Internal validity: Except for risk factor data, all variables assessed had high internal validity (>94\%) (Table 3). Fifty-seven percent of responses for risk factor in iPHIS did not match the paper-based chart. Of those records that did not match on medical risk factor, $46.3 \%$ had 'unknown' medical risk factor entered in iPHIS, whereas in the chart, the client's physician had specifically documented that there were no medical risk factors. In addition, $10.9 \%$ of the charts reviewed had a medical risk factor documented in the paper chart that was not entered in iPHIS.

Table 3: Internal validity of latent tuberculosis infection data fields $\left(\mathrm{N}=192^{1}\right)$

\begin{tabular}{|l|c|}
\hline \multicolumn{1}{|c|}{ Variable } & $\begin{array}{c}\text { Number (\%) of records with valid } \\
\text { data }\end{array}$ \\
\hline Gender & $191(99.5)$ \\
\hline Date of birth & $190(99.0)$ \\
\hline Treatment outcome status & $190(99.0)$ \\
\hline Reason treatment ended & $189(98.4)$ \\
\hline Immigration birth country & $188(97.9)$ \\
\hline Treatment end date & $185(96.4)$ \\
\hline Treatment start date & $181(94.3)$ \\
\hline Risk factor & $110(57.3)$ \\
\hline
\end{tabular}

Abbreviation: \%, percentage

${ }^{1}$ Total number of paper-based charts reviewed

External validity: Active follow-up by telephone with 208 LTBI cases that had no recorded treatment end date in iPHIS identified a treatment completion rate of $50 \%$. This is compared to treatment completion documented in iPHIS which was approximately 20\% from 2010 to 2013 (Table 4). In 2014, treatment completion for LTBI in iPHIS was $28 \%$, however, this includes clients that were part of the telephone survey where the treatment end date was subsequently entered into iPHIS. No new intervention was instituted that could account for the increase.

Table 4: External validity of latent tuberculosis infection treatment completion rates

\begin{tabular}{|c|r|r|}
\hline Year & $\begin{array}{c}\text { Total number of } \\
\text { cases that started } \\
\text { treatment }\end{array}$ & $\begin{array}{c}\text { Number (\%) of cases with } \\
\text { treatment completion in } \\
\text { iPHIS }\end{array}$ \\
\hline 2010 & 723 & $141(19.5)$ \\
\hline 2011 & 698 & $149(21.3)$ \\
\hline 2012 & 589 & $118(20.0)$ \\
\hline 2013 & 602 & $115(19.1)$ \\
\hline 2014 & 528 & $149(28.2)$ \\
\hline
\end{tabular}

Usefulness: The response rate for the key stakeholder interviews was $100 \%$. Overall, surveillance data were found to be useful for program planning and implementation (100\%). However, three themes emerged with some recommendations to improve usefulness of LTBI surveillance data:

1. Passive surveillance which relies on clinicians to submit completed forms is a barrier to completeness of information on risk factors and treatment completion. One interviewee stated that "data is as good (complete) as what we get from doctors...we see data gaps in risk factors and treatment completion". Electronic medical record alerts were suggested as one strategy to improve reporting among community clinicians. Another respondent elaborated on the consequences of incomplete data in terms of the ability to develop effective interventions for prevention and control. "(There are) gaps in physician reporting requirements and completion of the surveillance forms.... we don't know who to target and monitor more closely."

2. Surveillance data are used by PPH staff for public health action. One interviewee stated, "A surveillance report showed high rates of active TB and a low rate of reported LTBI in one municipality of Peel...so the TB nurses did physician outreach on who to screen to improve LTBI diagnosis" (in an effort to detect and treat LTBI cases to prevent progression to active TB).

3. Usefulness of LTBI surveillance data for program action can be refined with improved data analysis and collection of data specific to the local context. One interviewee asked, "How effective are our data in telling the LTBI story in Peel? Do Peel's active TB cases arise from known LTBI cases (where there was an opportunity to intervene)?" Another respondent stated that current policies for screening immigrants are resource intensive for "not a lot of transmission risk (in Peel); we need to tailor TB interventions to the local context."

\section{Discussion}

The evaluation of LTBI surveillance in Peel identified high data completeness (>99\%) for demographic variables, high data validity (>99\%) for most fields in iPHIS and LTBI treatment completion that is higher than what was documented in iPHIS. However, less than half of risk factor data in iPHIS is complete and just over half is valid, highlighting some of the limitations of the iPHIS system and the need to modify data entry and case management processes. Nonetheless, LTBI surveillance in Peel has proved useful for informing public health action, such as physician outreach interventions.

Several opportunities were identified to improve data quality. With $54.7 \%$ of LTBI risk factor data missing or 'unknown', next steps include standardizing processes for data entry. Because there is no field to capture 'no medical risk factors', this information was recorded in iPHIS as 'unknown' explaining in part the reason for this discordance. However, $10.9 \%$ of LTBI cases also had a documented medical risk factor in the paper chart that was not entered in iPHIS. The accuracy of risk factor data in iPHIS is particularly relevant from a cost-benefit perspective as resources could be mobilized to follow-up LTBI cases that are at increased risk of progressing to active TB (e.g. because of a medical risk factor). Valid and complete risk factor data are required to identify population groups at increased risk and in need of targeted measures.

The high percentage (64.6\%) of missing or 'unknown' treatment end date data is being addressed. The current passive physician reporting system contributes to the low percentage of recorded LTBI treatment completion. Active telephone follow-up of LTBI clients by public health staff better captures treatment 
completion data; however, with over 1,200 LTBI cases reported to Peel annually, this may not be sustainable. In the longer term, technology solutions that facilitate clinician reporting of risk factors and treatment completion to public health are being explored. Engaging community clinicians in an external stakeholder consultation would also be worthwhile to determine how to best address the challenges they face in reporting LTBI data.

One strength of this evaluation is that it provides direction to improve the usefulness of surveillance data, such as specific analyses to better understand the Peel LTBI population. For example, internal stakeholders discussed the public health role for management of LTBI detected during TB screening of new immigrants to Canada. The emergence of this theme from the interview data led Peel to re-analyze TB data from 2015, which identified that $23 \%$ of foreign-born TB cases are diagnosed within one to five years of immigrating to Canada, compared to $40 \%$ diagnosed with active TB after living in Canada for more than 15 years (unpublished). While this may be due to several reasons (e.g. development of chronic conditions which increase the risk of reactivation, or travel back to country of birth resulting in re-exposure to TB), the greatest risk for TB among Peel cases may not be at the time individuals immigrate to Canada when public health currently intervenes. Additional analyses to examine the differences between LTBI clients that complete treatment and those who do not is also underway.

Two limitations influence the evaluation results. Ideally, the assessment of external validity requires that surveillance data would be measured against a 'gold standard'. The authors of this article did not have access to LTBI patient records kept by community clinicians, therefore a telephone survey of LTBI cases to determine whether treatment was completed was used as the 'gold standard' comparison. While PPH telephone follow-up with a sample of LTBI cases indicated that treatment completion was under-reported in $\mathrm{PPHIS}$, the extent of under-reporting could not be quantified. However, there is also a risk that LTBI cases may not be aware that they did not complete the full nine months of treatment, so self-reporting on treatment completion may have been an overestimate. The second limitation was the inability to assess external completeness (or sensitivity), i.e., the extent to which Peel LTBI cases are captured by the current surveillance system. A study that evaluated LTBI surveillance in Massachusetts observed that substantial under-ascertainment of LTBI was likely and that mandatory reporting does not appear sufficient for LTBI detection (7). The authors suggest that enhanced targeted testing, active LTBI surveillance or laboratory-based surveillance may be needed to eliminate TB in the United States. These strategies may have applicability in Peel region as well.

LTBI surveillance data that are of high quality and useful for informing public health action are essential to TB prevention and control. Canadian TB standards target at least $80 \%$ treatment completion in LTBI cases that start treatment. In this evaluation telephone follow-up of Peel LTBI cases identified a treatment completion rate of $50 \%$, but this rate remains below the national target. While PPH continues active surveillance to ascertain true LTBI treatment completion rates, work to understand barriers to treatment initiation and completion in Peel region is also underway. It is anticipated that this surveillance evaluation will have relevance for other jurisdictions in Canada and other developed countries, particularly with sub-populations that have higher rates of active TB. Evaluating and improving LTBI surveillance is fundamental to advancing TB elimination efforts in Canada.

\section{Authors' statement}

JAM - conceptualization, methodology, formal analysis, investigation, writing-original draft, review and editing LF - conceptualization, writing-review and editing MV - conceptualization, methodology, writing-review and editing, supervision

\section{Conflict of interest}

None.

\section{Acknowledgements}

The authors would like to thank the community clinicians in Peel who reported LTBI data. From Peel Public Health, we thank the TB program staff, M Baltazar, S Raju, G Kujbida, M Horn, A Richards, R Strange, I Mogck, K Bingham and M Hau. From the Public Health Agency of Canada, we thank FW Tremblay, D Paquette, M Wood, N Gilbert, M McGuire, Field Service Training Response and Canadian Field Epidemiology Program Cohort 41.

\section{Funding}

This work was supported by the Public Health Agency of Canada's Canadian Field Epidemiology Program and Peel Public Health.

\section{References}

1. Public Health Agency of Canada, The Lung Association, Canadian Thoracic Society. Canadian Tuberculosis Standards, 7th edition [Internet]. Ottawa: Public Health Agency of Canada; 2014 [cited 2017 Feb 25]. Available from: www. respiratoryguidelines.ca/sites/all/files/Canadian_TB_ Standards_7th_Edition_ENG.pdf.

2. Kiazyk S, Ball TB. Latent tuberculosis infection: An overview. Can Commun Dis Rep. 2017;43(3):62-6. Available from: http://www.phac-aspc.gc.ca/publicat/ccdr-rmtc/17vol43/drrm43-3-4/ar-01-eng.php.

3. World Health Organization. Implementing the End TB Strategy: The essentials [Internet]. Geneva: WHO; 2015 [cited 2017 Mar 3]. Available from: www.who.int/tb/ publications/2015/The_Essentials_to_End_TB/en/.

4. Statistics Canada. National household survey 2011 [Internet]. Ottawa: Statistics Canada [updated 2011 Oct 05; cited 2017 Mar 15]. Available from: http://www23.statcan.gc.ca/imdb/ p2SV.pl?Function=getSurvey\&SDDS $=5178$.

5. Public Health Ontario. Reportable burdensome infectious disease incidence snapshot [Internet]. Tuberculosis Incidence. Toronto: Public Health Ontario [updated 2016 May 15; cited 
2017 Mar 15]. Available from: www.publichealthontario. ca/en/DataAndAnalytics/Snapshots/Pages/ReportableBurdensome-Infectious-Disease-Incidence.aspx.

6. Ontario Ministry of Health and Long-Term Care. Integrated Public Health Information System (iPHIS) database. Toronto: Government of Ontario; 2017. Data extracted by Peel Public Health, 2016 Mar 08. Further information available from: https://www.peelregion.ca/health/statusdata/DataSources/ HSD12_17.asp.

7. Hochberg N, Kubiak R, Tibbs A, Elder H, Sharnprapai S, Etkind S, Horsburgh Jr. C. Effectiveness of reporting on latent tuberculosis infection in Massachusetts, 2006-2008. Public Health Action. 2014 Mar 21;4(1):53-55.

8. Theocharopoulos G, Antoine D. Questioning the usefulness of the latent tuberculosis infection (LTBI) surveillance in children in France, 2010-2014. In: European Scientific Conference on Applied Infectious Disease Epidemiology abstract book. Stockholm: ECDC; 2016. p.112. Available from: http://ecdc.europa.eu/en/ESCAIDE/programme/ abstract-book/Documents/ESCAIDE\%20Abstract\%20 Book\%202016.pdf.
9. Sanderson J, Virkud A, Trieu L, Jacobson L, Crossa A, Ahuja $S$. Leveraging electronic health record data to evaluate the completeness of provider reporting for latent tuberculosis infection, New York City, 2013. Poster presentation: Council of State and Territorial Epidemiologists Annual Conference proceedings. June 2016. Available from: https://cste.confex. com/cste/2016/webprogram/start.html.

10. Government of Ontario. Health Protection and Promotion Act, R.S.O. 1990, c. H.7 [cited 2017 Mar 15]. Available from: www.ontario.ca/laws/statute/90h07.

11. Ministry of Health and Long Term Care. Ontario Public Health Standards: Tuberculosis prevention and control Protocol [Internet]. Toronto: Government of Ontario; 2008 [cited 2017 Feb 25]. Available from: www.health.gov. on.ca/en/pro/programs/publichealth/oph_standards/docs/ tuberculosis_prevention_control.pdf.

12. European Centre for Disease Prevention and Control. Technical document. Data quality monitoring and surveillance system evaluation [Internet]. Stockholm: ECDC; 2014 [cited 2017 Feb 25]. Available from: http://ecdc.europa. eu/en/publications/Publications/Data-quality-monitoringsurveillance-system-evaluation-Sept-2014.pdf.

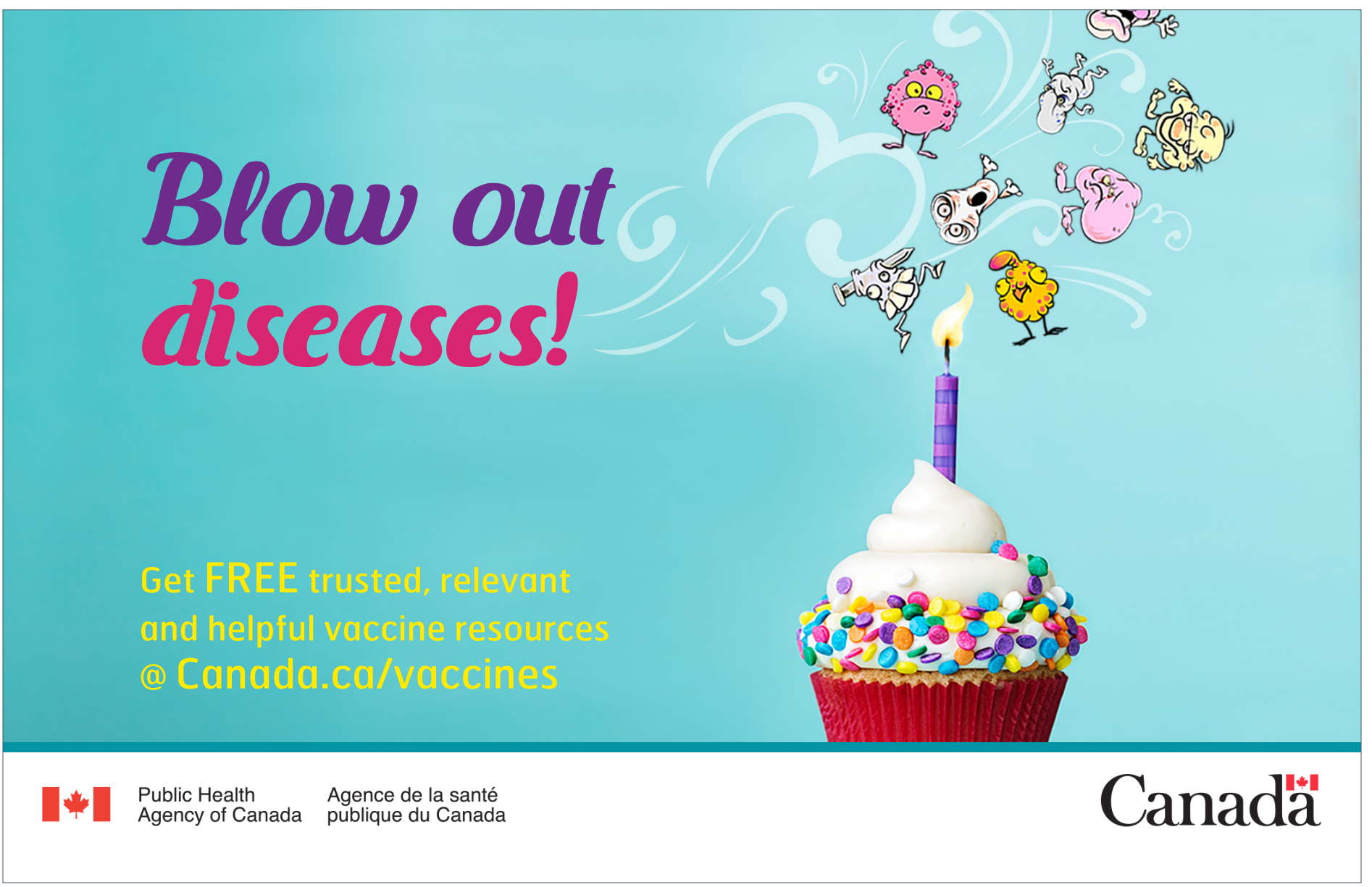

